\title{
Flexible Learning of Sparse Neural Networks via Constrained $L_{0}$ Regularization
}

Jose Gallego-Posada, Juan Ramirez and Akram Erraqabi

\section{Sparsity}

Training, storing and deploying NNs can be very expensive. Fortunately, their performance is robust to parameter pruning.

A method for obtaining efficient neural networks is by training them to encourage sparsity during training.

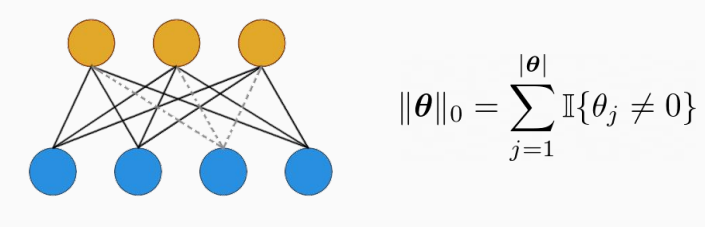

Regularization via $L_{0}$ penalties

(Louizos et al., 2018)

Penalize the number of active parameters.

$$
\mathcal{R}(\boldsymbol{\theta})=\frac{1}{N}\left(\sum_{i=1}^{N} \ell\left(h\left(x_{i} ; \boldsymbol{\theta}\right), y_{i}\right)\right)+\lambda\|\boldsymbol{\theta}\|_{0}
$$

Re-parametrize with differentiable stochastic gates based on concrete distributions.

$$
\underbrace{\boldsymbol{\theta}=\tilde{\boldsymbol{\theta}}}_{\begin{array}{c}
\text { free parameter } \\
\text { magnitudes }
\end{array}} \odot \underbrace{\boldsymbol{z}}_{\text {gates }}
$$

$\mathcal{R}(\tilde{\boldsymbol{\theta}}, \boldsymbol{\phi}) \triangleq \mathbb{E}_{\boldsymbol{z} \mid \boldsymbol{\phi}}[\mathcal{R}(\tilde{\boldsymbol{\theta}} \odot \boldsymbol{z})]$

$$
=\mathbb{E}_{\boldsymbol{z} \mid \boldsymbol{\phi}}\left[\frac{1}{N} \sum_{i=1}^{N} \ell\left(h\left(x_{i} ; \tilde{\boldsymbol{\theta}} \odot \boldsymbol{z}\right), y_{i}\right)\right]+\lambda \mathbb{E}_{\boldsymbol{z} \mid \boldsymbol{\phi}}\left[\|\boldsymbol{z}\|_{0}\right]
$$

\section{Regularization via $L_{0}$ constraints}

$$
\min _{\tilde{\boldsymbol{\theta}}, \boldsymbol{\phi}} \mathfrak{f}_{\text {obj }}(\tilde{\boldsymbol{\theta}}, \boldsymbol{\phi}) \triangleq \mathbb{E}_{\boldsymbol{z} \mid \boldsymbol{\phi}}\left[\frac{1}{N} \sum_{i=1}^{N} \ell\left(h\left(x_{i} ; \tilde{\boldsymbol{\theta}} \odot \boldsymbol{z}\right), y_{i}\right)\right]
$$

subject to $\quad \mathfrak{g}_{\text {const }}(\phi) \triangleq \mathbb{E}_{\boldsymbol{z} \mid \boldsymbol{\phi}}\left[\|\boldsymbol{z}\|_{0}\right] \leq \epsilon \cdot|\theta|$

$$
\begin{gathered}
\text { expected \# of } \\
\text { active params }
\end{gathered}
$$

Consider the associated Lagrangian and min-max game:

$$
\begin{array}{ll}
\mathcal{L}(\tilde{\boldsymbol{\theta}}, \boldsymbol{\phi}, \lambda) & \triangleq \mathfrak{f}_{\text {obj }}(\tilde{\boldsymbol{\theta}}, \boldsymbol{\phi})+\lambda\left(\frac{\mathfrak{g}_{\text {const }}(\boldsymbol{\phi})}{|\boldsymbol{\theta}|}-\epsilon\right) \\
\tilde{\boldsymbol{\theta}}^{*}, \boldsymbol{\phi}^{*}, \lambda^{*} & =\underset{\tilde{\boldsymbol{\theta}}, \boldsymbol{\phi}}{\operatorname{argmin}} \underset{\lambda \geq 0}{\operatorname{argmax}} \mathcal{L}(\tilde{\boldsymbol{\theta}}, \boldsymbol{\phi}, \lambda)
\end{array}
$$

\section{Constraints can be liberating}

- $\epsilon$ has straightforward semantics: the maximum proportion of active gates. Application specific requirements on sparsity can be incorporated into it.

$\Delta$ Tuning the penalization $\lambda$ to get a satisfactory model may require running several experiments. Even harder when introducing various sources of regularization.

$\Delta$ It is transparent whether a model is respecting the sparsity constraints.

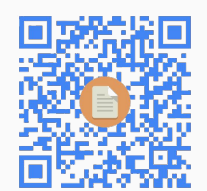

\section{Training dynamics}
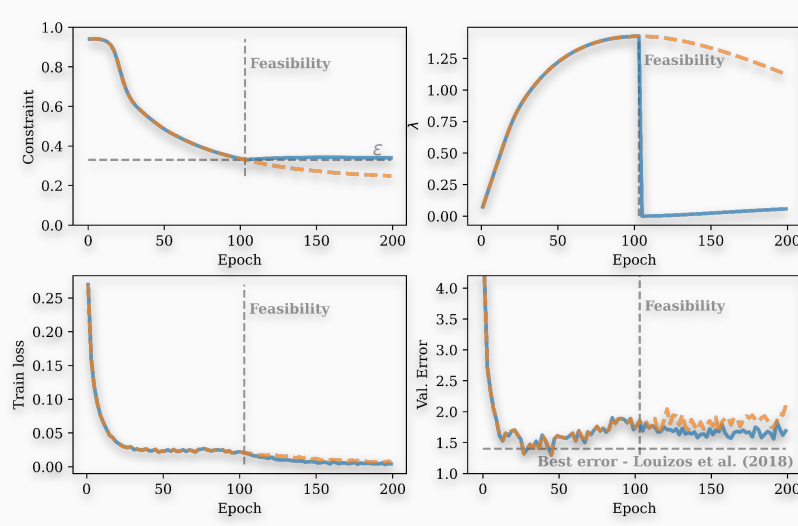

\section{Predictive performance}

\begin{tabular}{clcc}
\hline Architecture & Approach & Pruned & Best error (\%) \\
\hline MLP & Penalized $^{\dagger}: \lambda=0.1 / N$ & $219-214-100$ & 1.4 \\
\cline { 2 - 4 } 784-300-100 & Constrained: $\epsilon=33 \%^{198-233-100}$ & 1.4 \\
\hline \hline LeNet & Penalized $^{\dagger}: \lambda=0.1 / N$ & $20-25-45-462$ & 0.9 \\
\cline { 2 - 4 } 20-50-800-500 & Constrained: $\epsilon=10 \%^{20-21-34-407}$ & 0.53 \\
\hline
\end{tabular}

tC. Louizos, M. Welling, and D. P. Kingma. Learning Sparse Neural Networks through L0 Regularization. ICLR, 2018.

The constrained approach is not universally better than the penalized approach!

It can provide more flexibility and interpretability without compromising predictive performance. 